\title{
Molecular probes for the detection of pathogenic fungi in the presence of human tissue
}

\author{
R. KAPPE, C. N. OKEKE, C. FAUSER, M. MAIWALD and H.-G. SONNTAG \\ Hygiene-Institut, Im Neuenheimer Feld 324, D-69120 Heidelberg, University of Heidelberg, Germany
}

\begin{abstract}
Four primer systems, amplifying fragments of the gene coding for the small ribosomal subunit (18S rRNA) were characterised with pure cultures of 65 medically relevant fungal species plus two mushrooms. A primer cocktail (TR1/CA1-TR2/AF2) amplified 59 of 67 fungal species; the universal fungal primer 1 (UF1) in combination with the eukaryotic primers S3 or EU1 amplified 64 and 65 of 67 fungal species, respectively. The design of an additional primer (RZY1) enabled the amplification of the missing members of the zygomycetes. The primer systems amplified all the medically relevant fungi tested. These included eight Candida spp. and seven other yeast species, 13 dermatophytes, 32 moulds (including six zygomycetes and five dimorphic fungi) and two mushrooms. Eleven controls including DNA from Schistosoma mansoni, Escherichia coli, Mycobacterium tuberculosis and man were not amplified. The oligonucleotide CA hybridised with $C$. albicans, $C$. tropicalis and $C$. parapsilosis; the oligonucleotide TR hybridised with the 13 dermatophytes; the oligonucleotide AF hybridised with Aspergillus fumigatus, A. flavus, A. terreus, A. nidulans, A. versicolor, A. tamarii, A. clavatus, A. fischeri, but not with $A$. niger or $A$. versicolor; and the oligonucleotide HC hybridised with three varieties of Histoplasma capsulatum. These oligonucleotides did not hybridise with the other fungi nor the controls. The specificity of the newly designed primer systems was confirmed by selective amplification of fungal DNA from human lung tissue spiked with fungal biomass and from vitrectomy fluid of a patient with candida endophthalmitis.
\end{abstract}

\section{Introduction}

Deep-seated mycoses often present with non-specific symptoms and involve a large number of different organisms. Therefore, an appropriate diagnostic procedure will require a universal primary step of fungus detection for differentiation of a fungal infection from other infective diseases. This may be accomplished by PCR with universal fungal primers. A second general method should allow the identification of the causative agent, preferably to the species level. This may be accomplished by hybridisation with probes specific for common fungal species or fungal groups such as Candida or Aspergillus. To avoid the requirement of a wide range of specific antibodies or probes, sequence analysis of the amplification products may be preferred for rare agents of mycoses.

Received 16 May 1997; revised version accepted 16 Dec. 1997.

Corresponding author: Dr R. Kappe.
This proposed molecular approach would overcome several limitations of classical identification techniques: (1) slow growth of many relevant fungi with delayed production or lack of characteristic fruiting bodies or macroconidia; (2) the sexual reproduction cycle is unknown or lacking with many imperfect fungi; (3) special nutritional requirements of certain fungi; (4) similarity of macromorphology or micromorphology, or both, at the genus level; (5) antigenic cross-reactivity between important species and genera; and (6) possibly hazardous cultures for the health of laboratory personnel. In addition, problems originating in the availability of clinical samples and poor viability of fungal elements in smears or tissues may be overcome. There is a limited diversity of fungal growth in invasive disease. Branched hyphae with septae may be observed in tissue infected with Candida or Aspergillus spp. Distinct features, like fruiting bodies (e.g., Aspergillus heads) and characteristic conidia or spores (e.g., chlamydospores of $C$. albicans), are rarely produced in vivo. All classical identification techniques require considerable periods of time spent by specialised mycologically trained personnel. 
For the polymerase chain reaction (PCR)-mediated detection of fungi in clinical specimens, several primer systems, which do not cross-react with sequences of bacteria, parasites, plants, animals or man, have been described. Some are targeted to the gene coding for the small ribosomal subunit (18S rRNA), which consists of $c .1750 \mathrm{bp}$ [1-7]. Published primers have certain disadvantages, including gaps with important fungal pathogens like Cryptococcus and members of the class zygomycetes or amplification of rather large products $(\geqslant 500 \mathrm{bp})$ complicating the amplification from formalin-fixed tissue [1-7]. Sequences of the 18S rRNA gene are accessible for $>201$ fungi, including $>10$ yeast species and many other filamentous fungi of medical relevance [8-12]. Similar approaches have been undertaken which target the large subunit rRNA genes [13].

An earlier study reported the design and preliminary evaluation of fungal primer systems for 18S rDNA [6]. These quite universal fungal primer systems lacked cross-reactions with other eukaryotes, and amplified 10 representatives of the medically relevant fungal groups. However, possible gaps show up only in a large-scale evaluation.

The aims of this study were: (i) to evaluate published primers with an extended spectrum of 65 fungal pathogens and two mushrooms; (ii) to design a primer for pathogenic members of the zygomycetes, which failed to be amplified by any primer system published to date; (iii) evaluate published probes with an extended set of fungal amplicons and controls; and (iv) to show the utility of these molecular probes with human lung tissue and vitrectomy fluid. The oligonucleotides characterised in this study should enable the detection and preliminary classification of fungi in clinical specimens despite contamination with other eukaryotic cells, including human tissue, within a few days.

\section{Materials and methods}

\section{Primers and probes}

Database searches and sequence analyses were performed with the HUSAR (Heidelberg Unix Sequence Analysis Resources) computer system at the German Cancer Research Center, Heidelberg, Germany. The $18 \mathrm{~S}$ rDNA sequences of representative fungal species, plants and animals were retrieved from the European Molecular Biology Laboratory (EMBL, Heidelberg, Germany) database. Furthermore, the sequences of 201 fungal species were retrieved from the database available via Internet server URL: http:/rrna.uia.ac.be/.

The sequences were aligned with a multalign program [14] and visually checked for homologous regions, unique to fungi. The selected primers and probes
(Table 1) were custom synthesised by TIB Molbiol (Berlin, Germany).

\section{Organisms}

Sixty-five medically relevant fungal species, two mushroom species, nine eukaryotic controls, and two prokaryotic controls (Table 2) were selected for testing the specificity of the primer and probes. For testing of the primer system S1-RZY1 two additional members of the zygomycetes were included, Apophysomyces elegans and Syncephalastrum racemosum.

\section{Sample preparation}

Fungal and control DNA was extracted by microwave treatment, followed by a purification and concentration procedure with glass beads (Geneclean II Kit, Bio 101, USA).

Briefly, two loops of fungal biomass (yeast or mycelia) from several day- to several week-old cultures on Sabouraud's dextrose $(2 \%)$ agar were suspended in $350 \mu \mathrm{l}$ of TE buffer (Tris-HCl $10 \mathrm{mmol} / \mathrm{L}$, pH 8.0, Na-EDTA $1 \mathrm{mmol} / \mathrm{L}$ ) in $15-\mathrm{ml}$ plastic tubes and subjected to five cycles, $1 \mathrm{~min}$ each, of microwave treatment at $200 \mathrm{~W}$, with intermediate cooling on ice.

The Geneclean II kit was used according to the manufacturer's recommendations. Briefly, $300 \mu \mathrm{l}$ of the microwave-treated suspensions were transferred to 1.5-ml Eppendorf tubes containing $1 \mathrm{ml}$ of saturated $\mathrm{NaI}$ and $3 \mu \mathrm{l}$ of glass beads. The tubes were incubated at room temperature on a gyratory shaker at $100 \mathrm{rpm}$ for $15 \mathrm{~min}$, centrifuged at $17000 \mathrm{~g}$ for $1 \mathrm{~min}$ at $4^{\circ} \mathrm{C}$, and the supernate was discarded. The pellet was washed three times in $500 \mu \mathrm{l}$ of new wash buffer (contained in the Geneclean II kit). The final pellet was dried under vacuum, suspended in $50 \mu \mathrm{l}$ of water and incubated at $50^{\circ} \mathrm{C}$ for $5 \mathrm{~min}$. The DNA was recovered in the supernate following centrifugation and kept at $-20^{\circ} \mathrm{C}$ until used.

Human lung tissue was collected at autopsy from a patient who died from cardiac infarction. The tissue was free of contaminating fungi, as shown by culture of samples in Sabouraud's glucose broth. Lung tissue $(5 \mathrm{~g})$ was homogenised with $3 \mathrm{ml}$ of sterile $\mathrm{NaCl}$ solution with a stomacher (Lab Blender 80; Seward Medical UAC House, London). Samples $(200 \mu \mathrm{l})$ of lung homogenate were spiked with $100 \mu \mathrm{l}$ of a serial dilution of viable $C$. albicans in sterile distilled water giving final concentrations of $10^{8}-10^{0} \mathrm{ml}$ of lung tissue homogenate.

To study the effect of formalin fixation and embedding in paraffin wax, mycelia of $A$. fumigatus, Penicillium marneffei, Fusarium oxysporum and Pseudallescheria. boydii grown for 1 week on Sabouraud's glucose $(2 \%)$ agar, were fixed in formalin $4 \%$ overnight and 
embedded in paraffin wax. Five sections of $5 \mu \mathrm{m}$ each were transferred into a $1.5-\mathrm{ml}$ Eppendorf tube, deparaffinised with $n$-hexane, treated with ethanol $99.9 \%$ and subjected to the sample preparation method described above.

Finally, two samples of vitrectomy fluids from patients with endophthalmitis were examined; 3,6 and $9 \mu \mathrm{l}$ of the samples were subjected directly to the PCR without any pretreatment.

\section{PCR amplification}

PCR amplification was performed according to standard procedures [8] with the DNA extracted from fungi and control organisms and the following temperature cycling profile: initial denaturation at $95^{\circ} \mathrm{C}$ for $3 \mathrm{~min}$, followed by 35 cycles of denaturation at $95^{\circ} \mathrm{C}$ for $1 \mathrm{~min}$, annealing at $53^{\circ} \mathrm{C}$ for $1 \mathrm{~min}$ and extension at $72^{\circ} \mathrm{C}$ for $1 \mathrm{~min}$.

\section{Polyacrylamide gel electrophoresis and dot-blot hybridisation}

For detection of the amplified fragments, $30 \mu \mathrm{l}$ was removed from the reaction tubes and electrophoresed on a vertical polyacrylamide $5 \%$ gel with TBE buffer $(0.089 \mathrm{M}$ Tris, $0.089 \mathrm{M}$ boric acid, $0.002 \mathrm{M}$ EDTA). The gels were run at $12 \mathrm{~V} / \mathrm{cm}$ for $2 \mathrm{~h}$, stained with ethidium bromide in TBE for $15 \mathrm{~min}$ and visualised with a UV transilluminator. For dot-blotting, the DNA samples (PCR products) were heated to $95^{\circ} \mathrm{C}$ for $5 \mathrm{~min}$, chilled immediately on ice, and one volume of $20 \times \operatorname{SSC}(3 \mathrm{M} \mathrm{NaCl}, 0.3 \mathrm{M}$ sodium citrate) was added. The positively charged nylon membrane (Hybond ${ }^{\mathrm{TM}}$ $\mathrm{N}+$; Amersham Life Sciences) was pre-wetted with $10 \times \mathrm{SSC}$, secured in a microfiltration apparatus (BioDot ${ }^{\mathrm{K}} \mathrm{SF}$; BioRad Laboratories) and spotted with $2.0 \mu \mathrm{1}$ samples of the DNA, with drying time allowed between each sample. The membrane was incubated in a denaturing solution $(1.5 \mathrm{M} \mathrm{NaCl}, 0.5 \mathrm{M} \mathrm{NaOH})$ for $5 \mathrm{~min}$, and transferred to a filter paper wad soaked in neutralising solution $(1.5 \mathrm{M} \mathrm{NaCl}, 0.5 \mathrm{M}$ Tris- $\mathrm{HCl}$. $\mathrm{pH} 7.2,0.0001 \mathrm{M}$ EDTA) for $1 \mathrm{~min}$. The membrane was blot dried with filter paper and further dried in air. The DNA was fixed by baking the membrane in an oven at $80^{\circ} \mathrm{C}$ for $2 \mathrm{~h}$. The membrane was prehybridised at $48^{\circ} \mathrm{C}$ for $30 \mathrm{~min}$ in $0.9 \mathrm{M} \mathrm{NaCl}, 6 \mathrm{mM}$ EDTA, $90 \mathrm{mM}$ Tris, SDS $1 \%$, supplemented with yeast RNA (Boehringer, Mannheim, Germany) $1 \mathrm{~g} / \mathrm{L}$, followed by hybridisation at $43^{\circ} \mathrm{C}$ or $48^{\circ} \mathrm{C}$ overnight with ${ }^{32} \mathrm{P}$ labelled $\mathrm{CA}, \mathrm{AF}, \mathrm{TR}$ and $\mathrm{HC}$ oligonucleotides respectively in $15 \mathrm{ml}$ of prehybridisation solution. Membranes were washed in a solution of $3 \times \mathrm{SSC}$, SDS $5 \%$ and $10 \mathrm{~mm}$ sodium phosphate $(\mathrm{pH} \mathrm{7.0)}$ at $43^{\circ} \mathrm{C}$ or $48^{\circ} \mathrm{C}$ for $10 \mathrm{~min}$, then in $1 \times \mathrm{SSC}$, SDS $1 \%$ at $43^{\circ} \mathrm{C}$ or $48^{\circ} \mathrm{C}$, respectively, and exposed to a scientific imaging film (X-OMAT AR, Kodak, USA) in an X-ray film cassette.

\section{Results}

Twelve oligonucleotides, which have been described previously $[3,6,15]$ and the two new oligonucleotides CUF1 and RZY1, were custom synthesised (TIBMolbiol, Berlin, Germany). The primers were designated S1, S3, EU1, TR1, CA1, TR2, AF2, UF1, CUF1, RZY1 and the ${ }^{32} \mathrm{P}$-labelled hybridisation oligonucleotides CA, AF, TR, and HC (Table 1). The primers have different specificities and amplify 18S rDNA-fragments of lengths presented in Fig. 1.

To rule out possible non-specific inhibition of the PCR reactions by constituents of the samples, control reactions with the broadly reactive primers $\mathrm{S} 1$ and S3 for eukaryotic 18S rDNA [16] were performed. Under identical PCR conditions, these control primers amplified the DNA of all 67 fungi along with the DNA of nine eukaryotic control organisms, but not the DNA of the prokaryotes Escherichia coli and Mycobacterium tuberculosis (Table 2). Seventy-six amplicons had the expected size, and two were larger than expected by c. $400 \mathrm{bp}$. These two (Exophiala) Wangiella dermatitidis and Histoplasma capsulatum var. farciminosum) contained introns, proven by sequence analysis (data not shown).

In a first attempt to amplify the $18 \mathrm{~S}$ rDNA of a larger spectrum of fungal species than described previously [1-5], the dermatophyte specific primer system TR1TR2 [3] was supplemented with the candida primer CA1 and the aspergillus primer AF2: The resulting primer cocktail (TR1/CA1-TR2/AF2) amplified the DNA of 59 fungal species and the amplicons obtained had the expected fragment lengths with the exception of $H$. capsulatum var. farciminosum. This primer cocktail failed to amplify Rhodotorula, Malassezia, Exophiala/Wangiella, Phialophora, Rhizopus, Syncephalastrum and the mushrooms. With the exception of the plants Physcomitrella, Abies and Brassica, the controls were either not amplified or produced amplicons of clearly different sizes (Table 2). Polyacrylamide gels demonstrating the clearly different sizes of the $E$. coli amplicons have been published previously [6].

The primer system UF1-S3 was designed to reduce the number of primers involved, to further increase the number of fungal species covered and to reduce the number of cross-reacting controls. It amplified the DNA of 59 representative fungal species and the amplicons obtained had the expected fragment lengths. The zygomycetes Rhizopus, Rhizomucor and Absidia were not amplified. With the exception of the prokaryote $E$. coli, which produced an amplicon of a clearly different size, and the plants Physcomitrella and Brassica, which cross-reacted, none of the controls was amplified (Table 2).

The reverse primer EU1 was designed to enable the 


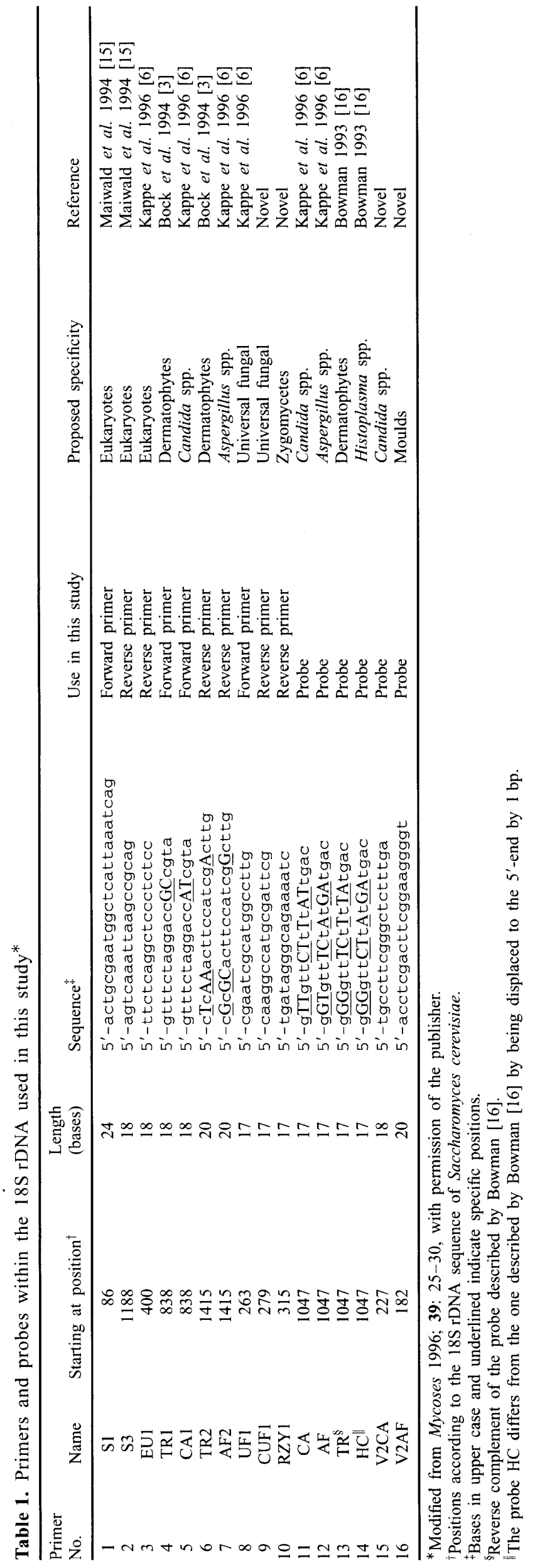




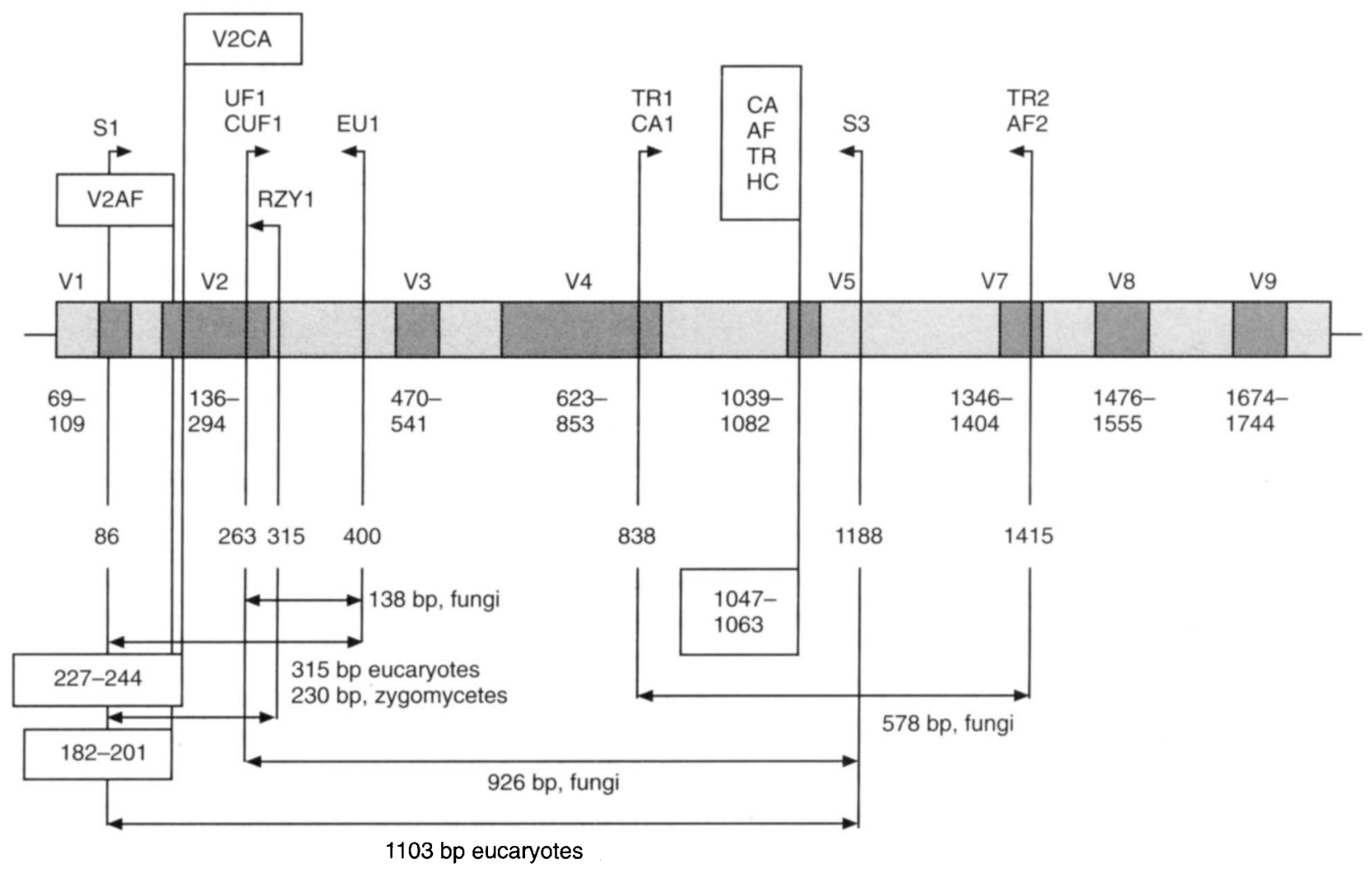

Fig. 1. (Modified from Mycoses 1996; 39: 25-30, with permission of the publisher). Scheme of the gene encoding the eukaryotic small ribosomal subunit (18S rDNA) with highly conserved regions (light grey) and variable regions V1-V9 (dark grey, adapted from [12]) (V6 exists in prokaryotes only). Positions of primers, amplicons and probes used in this study are indicated. S1, UF1, TR1 and CA1 are forward primers; CUF1, RZY1, EU1, S3, TR2 and AF2 are reverse primers. CA, AF, TR and HC (framed) denote group-specific hybridisation oligonucleotides. Numbers indicate starting and ending positions of the variable regions V1-V9 and of the hybridisation oligonucleotides, and starting positions of primers according to the sequence of Saccharomyces cerevisiae. Amplification products are labelled with lengths in basepairs and proposed specificity.

amplification of shorter products in combination with S1 or with UF1, which may be an advantage for certain applications, e.g., PCR amplification from paraffinembedded tissues [17]. The amplification spectrum and the specificity of the primer system UF1-EU1 was similar to that of UF1-S3, except that the former also weakly amplified Rhizomucor and cross-reacted with Abies lasiocarpa (Table 2). Expectedly, the presence of introns in the $18 \mathrm{~S}$ rDNA of $H$. capsulatum var. farciminosum and $E . / W$. dermatitidis was not revealed in the UF1-EU1 amplicons, as the intron insertion positions lie outside the amplified regions. To cover the complete spectrum of the zygomycetes, the reverse primer RZY1 was designed, starting at position 315 , at the end of the variable region 2 (V2) near the position of UF1 and the reverse primer CUF1 (complement of UF1) (Table 1, Fig. 1). The combination of RZY1 and S1 enabled the amplification of $230-b p$ products of eight members of the zygomycetes (Abs. corymbifera, Apo. elegans, Mucor circinelloides, Rhizomucor pusillus, Rhizopus oryzae, Saksenaea vasiformis, Cunninghamella bertholletiae and Syn. racemosum), and there was no amplification of any of the other 72 organisms presented in Table 2 .
The fungal origin of the amplification products as well as a preliminary taxonomic classification were revealed by hybridisation. The oligonucleotide $\mathrm{CA}$ reacted with the amplicons from $C$. albicans, $C$. tropicalis and $C$. parapsilosis, but not with the other fungi nor the controls (Fig. 2b). The oligonucleotide AF reacted with the amplicons from $A$. fumigatus, $A$. flavus, $A$. terreus, $A$. versicolor (weak), A. tamarii, $A$. clavatus and $A$. fischeri, but not with the other fungi or the controls (Fig. 2c). The oligonucleotide TR reacted with the amplicons from all 13 dermatophytes tested (Fig. 2d), including Microsporum ferrugineum (not shown), but not with those of the other fungi or the controls. The oligonucleotide $\mathrm{HC}$ reacted with the amplicons from $H$. capsulatum var. capsulatum, $H$. capsulatum var. duboisii and $H$. capsulatum var. farciminosum, but not with those of other fungi or the controls (Fig. 2e).

To prove the suitability of the primer systems for amplification of fungal DNA from clinical samples, human lung tissue was spiked with $C$. albicans and subjected to sample preparation and PCR. Selective amplification of $C$. albicans DNA was possible with 


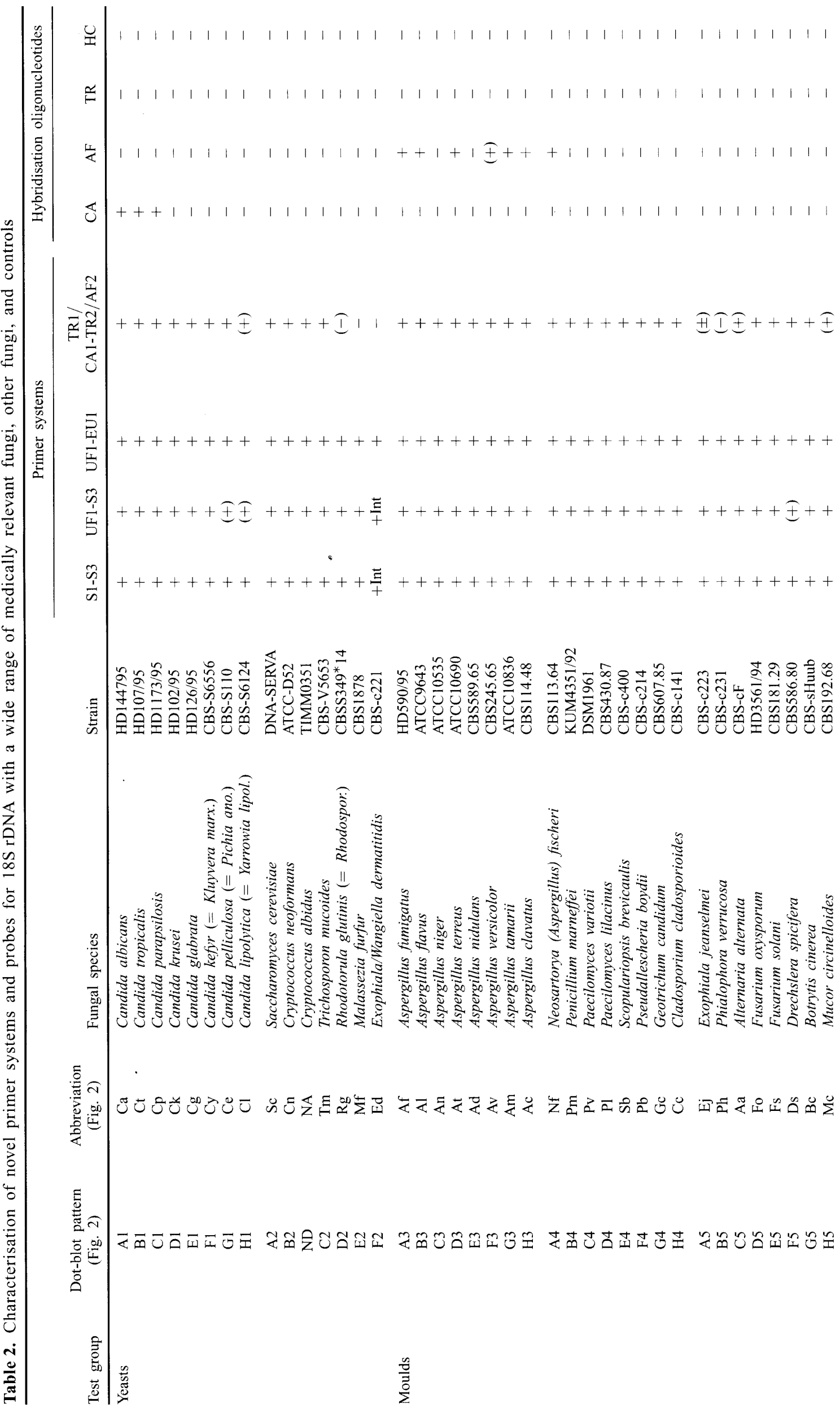




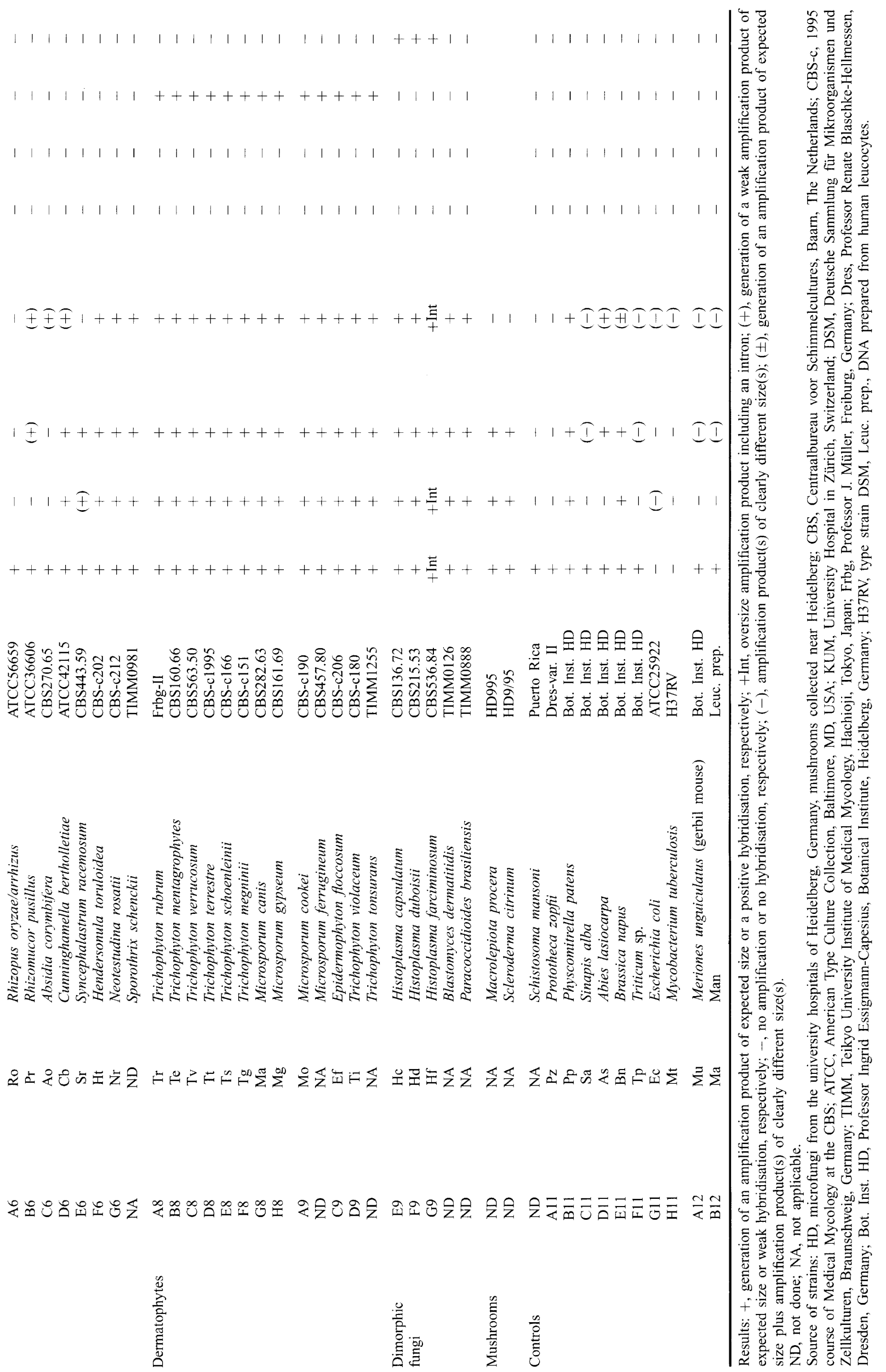


$\begin{array}{llllllllllll}1 & 2 & 3 & 4 & 5 & 6 & 7 & 8 & 9 & 10 & 11 & 12\end{array}$

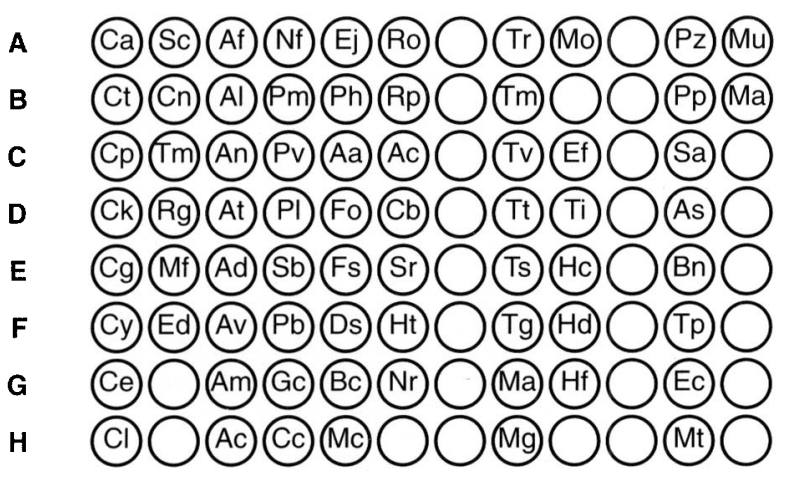

(a)
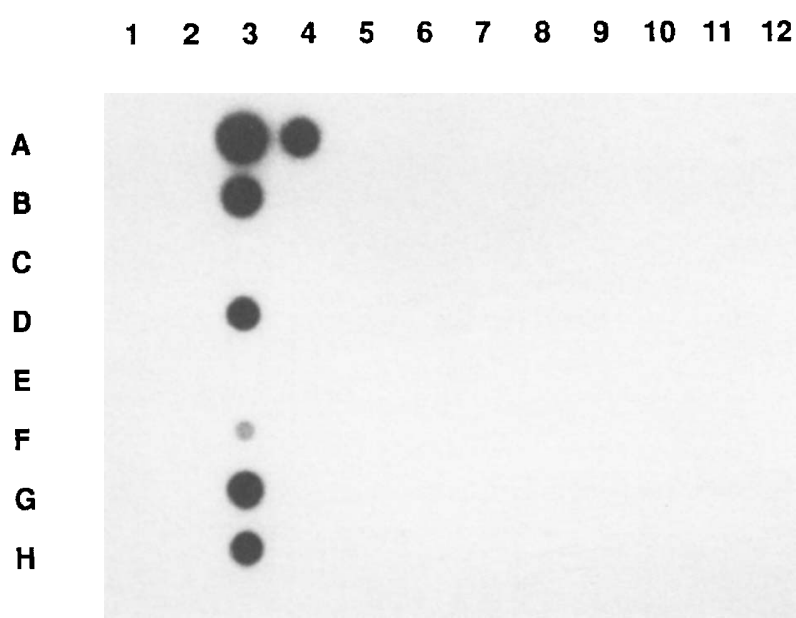

(c)

\section{$\begin{array}{llllllllllll}1 & 2 & 3 & 4 & 5 & 6 & 7 & 8 & 9 & 10 & 11 & 12\end{array}$}

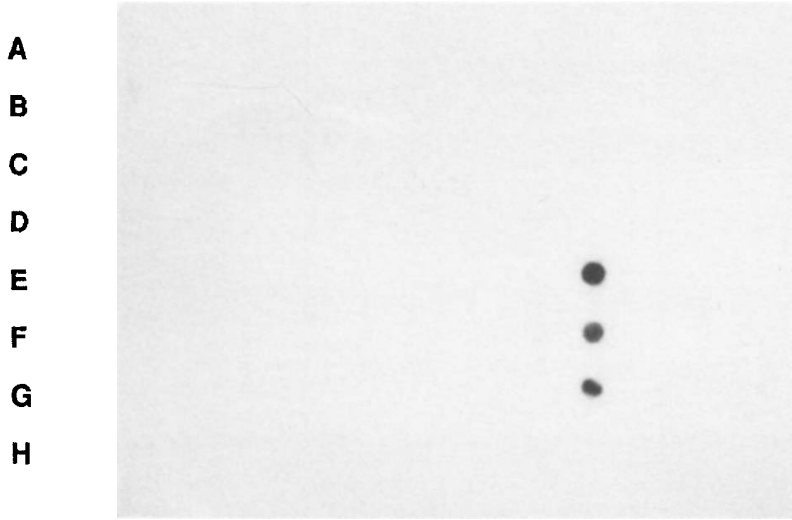

(e)

the primers S1-CUF1 with a level of sensitivity of $10^{6}$ C. albicans blastospores $/ \mathrm{ml}$ of homogenised lung tissue.

Formalin fixation and paraffin embedding of fungal mycelia did not prevent amplification of small products. Positive results were obtained with $A$. fumigatus, $P$. marneffei, $F$. oxysporum and $P$. boydii with the primer pairs UF1-EU1 (138-bp product) and S1-CUF1 (178-bp product) in three of three experiments and four of four experiments, respectively. $\begin{array}{llllllllllll}1 & 2 & 3 & 4 & 5 & 6 & 7 & 8 & 9 & 10 & 11 & 12\end{array}$

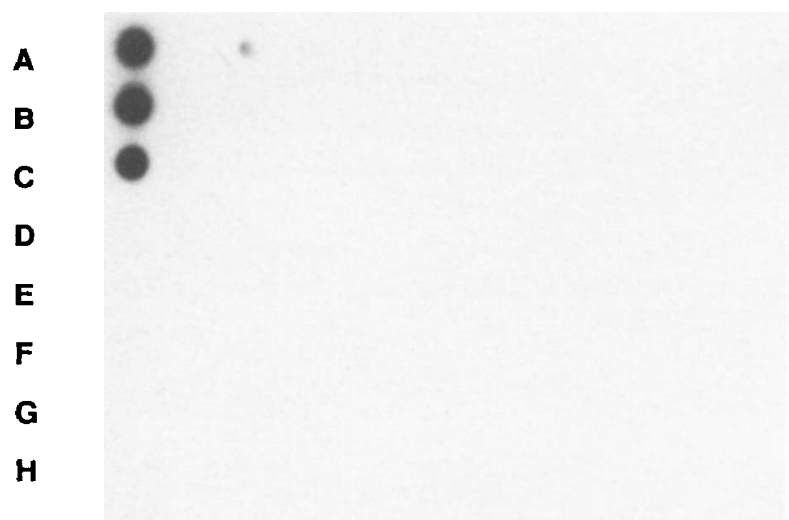

(b)

$$
\begin{array}{llllllllllll}
1 & 2 & 3 & 4 & 5 & 6 & 7 & 8 & 9 & 10 & 11 & 12
\end{array}
$$

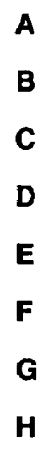

(d)

Fig. 2. Dot-blot and hybridisation patterns of 59 representative fungal species and 10 controls. The nylon membrane was spotted according to the pattern presented in panel a. Abbreviations as in Table 2. Panels b-e represent results of hybridisations with the probes $\mathrm{CA}$, $\mathrm{AF}, \mathrm{TR}$ and HC, respectively. The $69 \mathrm{PCR}$ products were amplified by the primer systems UF1-S3 or S1-S3. The membrane was separately hybridised with each probe, i.e., autoradiographed and stripped after each hybridisation.

The results of assay of the vitrectomy fluids are presented in Fig. 3. The patient with candida endophthalmitis was clearly positive, whereas the other patient with endophthalmitis of unknown aetiology and the controls were negative.

\section{Discussion}

As there is no $18 \mathrm{~S}$ rDNA sequence variation within a given species (no strain variation) and results matched 


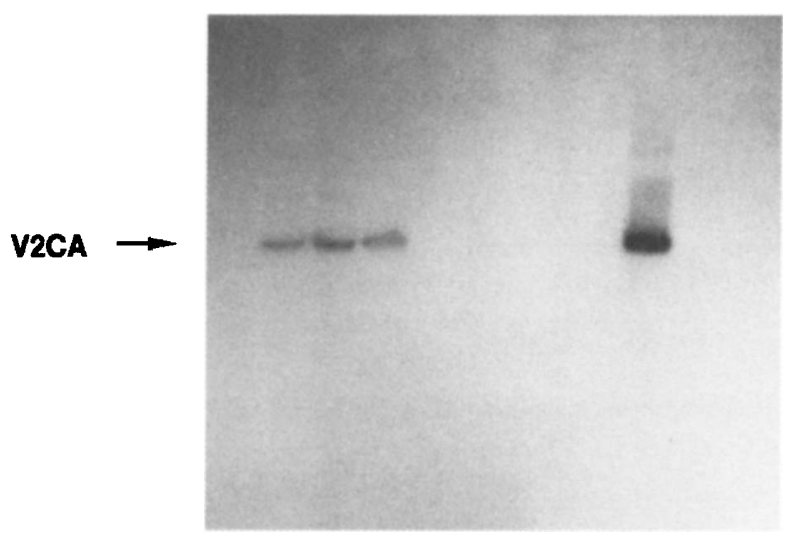

Fig. 3. Amplification of native vitrectomy fluid (VF) of two patients with endophthalmitis (primers S1-CUF1, $178 \mathrm{bp}$ of $18 \mathrm{~S}$ rDNA) and hybridisation with the Candidaspecific probe V2CA. Patient 1 was suffering from candida endophthalmitis, patient 2 had an endophthalmitis of unknown aetiology. Lanes: 1, 100-bp ladder; 2, $3 \mu \mathrm{l}$ of VF patient $1 ; 3,6 \mu \mathrm{l}$ of VF patient $1 ; 4,9 \mu 1$ of VF of patient $1 ; 5,3 \mu \mathrm{l}$ of VF of patient $2 ; 6,6 \mu 1$ of VF of patient $2 ; 7,9 \mu \mathrm{l}$ of VF of patient $2 ; \mathbf{8}$, not loaded; $\mathbf{9}$, positive control; 10, not loaded; 11, negative control.

those expected from published sequences [12], there was no need to study multiple strains of each species.

The primer systems TR1/CA1-TR2/AF2 and UF1-S3 amplified DNA from 59 and 64 representatives of the medically relevant fungal groups, respectively (yeasts, moulds, dermatophytes and dimorphic fungi), but not the DNA of other eukaryotic organisms, including the parasite Schistosoma mansoni, the yeast-like alga Pro. zopfii and man (Table 2). The failure to react with controls was not due to the lack of target DNA nor to an inhibition of the PCR, because broadly reactive eukaryotic primers readily amplified all samples tested except the prokaryotes $E$. coli and $M$. tuberculosis (Table 2). The primer UF1 failed to amplify some members of the zygomycetes, including Rhizopus oryzae and Abs. corymbifera, and was not a truly 'universal fungal' primer in this study. Weak amplification or complete failure to amplify a fungal species may be explained by the number and the position of nucleotide mismatches of the primers to the fungal DNA. The failure of UF1 to amplify some of the zygomycetes is partially explained by sequence information on the 18S rDNA of Rhizomucor pusillus, Rhizopus oryzae and Abs. corymbifera (personal communication, K. O'Donnell, and own unpublished data), which differ considerably from $18 \mathrm{~S}$ rDNA sequences of basidiomycetes and ascomycetes. Therefore, for a complete amplification of the order Mucorales, with the $18 \mathrm{~S}$ rDNA, the additional oligonucleotide RZY1, was designed, which is homologous for the eight zygomycetes studied. As its position on the $18 \mathrm{~S}$ rDNA is very close to UF1 and CUF1, a single truly universal fungal PCR may be developed, which could work with one broadly reactive forward primer at the beginning of the $18 \mathrm{~S}$ rDNA and a combination of CUF1 and RZY1 (at the end of the variable region $\mathrm{V} 2$, around position 280). Advantages of this asymmetrical PCR would include a short amplification product (c. $200 \mathrm{bp}$ ), which contains the variable region $\mathrm{V} 2$ for specification.

Weak hybridisation or complete failure of a probe to hybridise with a fungal amplicon is explained by at least one nucleotide mismatch of the hybridisation oligonucleotide to the corresponding sequence of the fungal amplicon. Published fungal sequences [9-11] allow the following reasoning. The CA probe perfectly fits C. albicans, C. tropicalis and C. parapsilosis; however, it contains mismatches for $C$. krusei $(\mathrm{n}=10), C$. glabrata $(\mathrm{n}=6), C$. kefyr $(\mathrm{n}=6), C$. pelliculosa $(\mathrm{n}=7), C$. lipolytica $(\mathrm{n}=12)$. The $\mathrm{AF}$ probe perfectly fits A. fumigatus, A. flavus, A. terreus, A. tamarii and $N$. (A.) fischeri; however, it contains mismatches for $A$. niger $(\mathrm{n}=1)$ and $A$. nidulans $(\mathrm{n}=3)$. The TR probe perfectly fits all dermatophytes, but it contains mismatches for all other fungi. The HC probe perfectly fits the three Histoplasma varieties, but contains mismatches for all other fungi. For identification of fungal amplification products that fail to hybridise with the probes mentioned, additional probes may be designed. However, direct sequence analysis is recommended for rare agents of mycotic infections.

The 18S rRNA gene was used for this study, because of the large number of known sequence [9-11] and the favourable relationship of conserved and variable regions (V1-V9, Fig. 1), which allow the design of suitable multipurpose oligonucleotides.

Other primer systems within the gene coding for the $18 \mathrm{~S}$ rRNA have been described as fungus-specific [1, $2,4,5,7]$. However, these primers, which have been characterised with a limited number of fungi, failed to amplify several species of medically important genera including Alternaria, Cryptococcus, Mucor and Sporothrix, because of three or more mismatches in each case.

PCR-based detection and identification of fungi has been described for several types of clinical specimens including samples from the respiratory tract [18-21] and serum or blood [2, 7, 22-25]. Claimed sensitivities of $1 \mathrm{cfu}$ of $C$. albicans $/ \mathrm{ml}$ of blood [2, 7] have not been confirmed. Sample preparation remains a serious problem, limiting the sensitivity of the assays.

Identification of fungi in paraffin-embedded tissue has been achieved by immunohistochemistry [26], by insitu hybridisation [27] and by sequencing of ribosomal DNA [28]. Immunohistochemistry and in-situ hybridisation require specific probes and do not lead to species identification. Formaldehyde fixation appears to modify human and viral DNA $[17,28]$. Williams et 
al. [28] amplified candida DNA fragments (218 bp) from paraffin wax-embedded tissue. The preliminary experiments described in the present study indicate that selective amplification of fungal DNA from human lung tissue spiked with $C$. albicans is possible with short products (138 and $178 \mathrm{bp}$ ) with a sensitivity of $10^{6}$ C. albicans cells $/ \mathrm{ml}$ of homogenised lung tissue. Furthermore, amplification was still possible after formalin fixation and embedding in paraffin of important pathogens, which are hard to distinguish by histology. Finally, the results with the vitrectomy fluids show that the primer systems evaluated in this study work with clinical samples. The simplified strategy for clinical samples will include PCR amplification by broadly reactive fungal primers followed by hybridisation with probes for the most common fungal pathogens, i.e. Candida spp. (V2CA specific for $C$. albicans or C. parapsilosis) or Aspergillus spp. (V2AF specific for moulds, but lacking cross-reactions with yeasts). Amplification products of rare fungal species should be sequenced for species identification rather than probing with a huge set of species-specific primers, which could never be exhaustive.

This work was supported in part by a grant from the Deutschsprachige Mykologische Gesellschaft, Germany (R. K.) and by a fellowship from the Alexander von Humboldt-Stiftung, Germany $(\mathrm{C}$. O.). We thank O. Nolte and H-J. Ditton (Hygiene-Institut, Heidelberg) for technical advice and support, and Professor Esther Segal for critical reading of the manuscript.

\section{References}

1. White TJ, Bruns T, Lee S, Taylor J. Amplification and direct sequencing of fungal ribosomal RNA genes for phylogenetics. In: Innis MA, Gelfand DH, Sninsky JJ, White TJ (eds) PCR protocols: a guide to methods and applications. San Diego, CA, Academic Press. 1990: 315-322.

2. Polanco AM, Rodríguez-Tudela JL. Martínez-Suárez JV. Detection of pathogenic fungi in human blood by the polymerase chain reaction. Eur $J$ Clin Microbiol Infect Dis 1995; 14: 618-621.

3. Bock M, Maiwald M, Kappe R, Nickel P, Näher H. Polymerase chain reaction-based detection of dermatophyte DNA with a fungus-specific primer system. Mycoses 1994; 37: 79-84.

4. Makimura K, Murayama SY, Yamaguchi, H. Detection of a wide range of medically important fungi by the polymerase chain reaction. J Med Microbiol 1994; 40: 358-364.

5. Makimura K, Murayama SY, Yamaguchi, H. Specific detection of Aspergillus and Penicillium species from respiratory specimens by polymerase chain reaction (PCR). Jpn J Med Sci Biol 1994; 47: 141-156.

6. Kappe R, Fauser C, Okeke CN, Maiwald M. Universal fungus specific primer systems and group-specific hybridization oligonucleotides for 18S rDNA. Mycoses 1996; 39: 25-30.

7. Einsele $H$, Hebart $H$, Roller $G$ et al. Detection and identification of fungal pathogens in blood by using molecular probes. J Clin Microbiol 1997; 35: 1353-1360.

8. Mitchell TG, Sandin RL, Bowman BH, Meyer W, Merz WG. Molecular mycology: DNA probes and applications of PCR technology. J Med Vet Mycol 1994; 32 Suppl 1: 351-366.

9. Neefs J-M, Van de Peer Y, De Rijk P, Chapelle S, De Wachter
R. Compilation of small ribosomal subunit RNA structures. Nucleic Acids Res 1993; 21: 3025-3049.

10. Van de Peer Y, Van den Broeck I, De Rijk P, De Wachter R. Database on the structure of small ribosomal subunit RNA. Nucleic Acids Res 1994; 22: 3488-3494.

11. Van de Peer, Nicolai S, De Rijk P, De Wachter R. Database on the structure of small ribosomal subunit RNA. Nucleic Acids Res 1996; 24: 86-91.

12. Verweij PE, Meis JFGM, van den Hurk P, Zoll J, Samson RA, Melchers WJG. Phylogenetic relationships of five species of Aspergillus and related taxa as deduced by comparison of sequences of small subunit ribosomal RNA. J Med Vet Mycol 1995; 33: $185-190$

13. Sandhu GS, Kline BC, Stockman L, Roberts GD. Molecular probes for diagnosis of fungal infections. J Clin Microbiol 1995; 33: 2913-2919.

14. Krüger M, Osterburg G. On the alignment of two or more molecular sequences. Computer Programs in Biomedicine 1983; 16: $68-69$.

15. Maiwald M, Kappe R, Sonntag H-G. Rapid presumptive identification of medically relevant yeasts to the species level by polymerase chain reaction and restriction enzyme analysis. $J$ Med Vet Mycol 1994; 32: 115-122.

16. Bowman $\mathrm{BH}$. A model PCR/probe system for the identification of fungal pathogens. In: Persing DH, Smith TF, Tenover FC, White TJ (eds) Diagnostic molecular microbiology, principles and applications. Washington, DC, American Society for Microbiology. 1993: 423-430.

17. Karlsen F, Kalantari M, Chitemerere M, Johansson B, Hagmar B. Modifications of human and viral deoxyribonucleic acid by formaldehyde fixation. Lab Invest 1994; 71: 604-611.

18. Olsson M, Elvin K, Löfdahl S, Linder E. Detection of Pneumocystis carinii DNA in sputum and bronchoalveolar lavage samples by polymerase chain reaction. J Clin Microbiol 1993; 31: $221-226$

19. Spreadbury C, Holden D, Aufauvre-Brown A, Bainbridge B, Cohen J. Detection of Aspergillus fumigatus by polymerase chain reaction. J Clin Microbiol 1993; 31: 615-621.

20. Montone K, Litzky LA. Rapid method for detection of Aspergillus 5S ribosomal RNA using a genus-specific oligonucleotide probe. Am J Clin Pathol 1995; 103: 48-51.

21. Bretagne S, Costa J-M, Marmorat-Khuong A et al. Detection of Aspergillus species DNA in bronchoalveolar lavage samples by competitive PCR. J Clin Microbiol 1995; 33: 1164-1168.

22. Miyakawa Y, Mabuchi T, Kagaya K, Fukazawa Y. Isolation and characterization of a species-specific DNA fragment for detection of Candida albicans by polymerase chain reaction. $J$ Clin Microbiol 1992; 30: 894-900.

23. Chryssanthou E, Andersson B, Petrini B, Löfdahl S, Tollemar J. Detection of Candida albicans DNA in serum by polymerase chain reaction. Scand J Infect Dis 1994; 26: 479-485.

24. Holmes AR, Cannon RD, Shepherd MG, Jenkinson HF. Detection of Candida albicans and other yeasts in blood by PCR. J Clin Microbiol 1994; 32: 228-231.

25. Rand $\mathrm{KH}$, Houck $\mathrm{H}$, Wolff $\mathrm{M}$. Detection of candidemia by polymerase chain reaction. Mol Cell Probes 1994; 82: 215-221.

26. Monteagudo C, Marcilla A, Mormeneo S, Llombart-Bosch A Sentandreu R. Specific immunohistochemical identification of Candida albicans in paraffin-embedded tissue with a new monoclonal antibody (1B12). Am J Clin Pathol 1995; 103: $130-135$.

27. Lischewski A, Amann RI, Harmsen D, Merkert H, Hacker J Morschhäuser J. Specific detection of Candida albicans and Candida tropicalis by fluorescent in situ hybridization with an 18S rRNA-targeted oligonucleotide probe. Microbiology 1996; 142: $2731-2740$

28. Williams DW, Wilson MJ, Lewis MAO, Potts AJC. Identification of Candida species in formalin fixed, paraffin wax embedded oral mucosa by sequencing of ribosomal DNA. $J$ Clin Pathol: Mol Pathol 1996; 49: M23-M28. 